\title{
Multi-scale analysis of teleconnection indices: climate noise and nonlinear trend analysis
}

\author{
C. Franzke \\ British Antarctic Survey, Natural Environment Research Council, Cambridge, UK \\ Received: 13 October 2008 - Revised: 8 January 2009 - Accepted: 9 January 2009 - Published: 6 February 2009
}

\begin{abstract}
The multi-scale nature and climate noise properties of teleconnection indices are examined by using the Empirical Mode Decomposition (EMD) procedure. The EMD procedure allows for the analysis of non-stationary time series to extract physically meaningful intrinsic mode functions (IMF) and nonlinear trends. The climatologically relevant monthly mean teleconnection indices of the North Atlantic Oscillation (NAO), the North Pacific index (NP) and the Southern Annular Mode (SAM) are analyzed.

The significance of IMFs and trends are tested against the null hypothesis of climate noise. The analysis of surrogate monthly mean time series from a red noise process shows that the EMD procedure is effectively a dyadic filter bank and the IMFs (except the first IMF) are nearly Gaussian distributed. The distribution of the variance contained in IMFs of an ensemble of AR(1) simulations is nearly $\chi^{2}$ distributed. To test the statistical significance of the IMFs of the teleconnection indices and their nonlinear trends we utilize an ensemble of corresponding monthly averaged AR(1) processes, which we refer to as climate noise. Our results indicate that most of the interannual and decadal variability of the analysed teleconnection indices cannot be distinguished from climate noise. The NP and SAM indices have significant nonlinear trends, while the NAO has no significant trend when tested against a climate noise hypothesis.
\end{abstract}

\section{Introduction}

The analysis of climate time series provides insight for understanding and predicting climate variability. An important topic in climate research is the existence of dynamically relevant and statistically significant modes of variability and

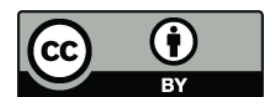

\section{Correspondence to: C. Franzke} (chan1@bas.ac.uk) trends. Most of the atmospheric mid-latitude variability can be described by just a few large-scale teleconnection patterns (e.g. Wallace and Gutzler, 1981) which explain most of the variance and exert a huge influence on regional surface climate and seasonal climate conditions. The most pronounced patterns in the Northern Hemisphere are the North Atlantic Oscillation (NAO) and the Pacific-North America (PNA) pattern whose surface imprint is also known as the North Pacific (NP) index. The Southern Hemisphere is dominated by the Southern Annular Mode (SAM). Therefore, the study of univariate teleconnection indices provides insight into climate dynamics and global climate change.

Time series from nature are in general nonlinear and nonstationary. Most traditional time series analysis methods are only valid when applied to stationary time series. See Huang et al. (1998) for an extensive review of time series analysis methods. The recently developed Empirical Mode Decomposition method (Huang et al., 1998; Huang and Wu, 2008) is able to extract physically meaningful modes from time series which are both non-stationary and nonlinear. This method has been widely and successfully applied in climate science (e.g. Duffy, 2004; McDonald et al., 2007; Wu et al., 2007; Huang and $\mathrm{Wu}, 2008$ ).

The climate system is undoubtedly a multi-scale system where a multitude of vastly different time and space scales nonlinearly interact with each other. The climate variability on long time scales is usually investigated by examining averaged data (monthly or seasonal means), though the intrinsic time scale of most teleconnection patterns is about 10 days (Feldstein, 2000a; Franzke and Feldstein, 2005). Thus, it is possible that part of the observed climate variability on monthly and seasonal time scales stems from the fast weather fluctuations as a result of the averaging. This part of the variability is called climate noise (Leith, 1973; Madden, 1976, 1981; Feldstein, 2000a,b; Czaja et al., 2003). While this part can be thought of stemming from processes intrinsic to the atmosphere, variability on longer time scales is usually thought

Published by Copernicus Publications on behalf of the European Geosciences Union and the American Geophysical Union. 
as being caused by external (not atmospheric) processes like the sea surface temperature, sea ice and solar insolation. For this reason it is necessary to identify which part of observed climate variability most likely stems from intrinsic weather fluctuations and which part most likely stems from external processes. One null hypothesis for climate variability on seasonal and longer time scales is that climate variability can be explained as the integral response to random weather fluctuations which occur on time scales of just a few days (Hasselmann, 1976). The paradigmatic model in climate science for such a process is a first order Markov process (represented by an autoregressive process of first order $\operatorname{AR}(1)$ ). The power spectrum of such a process is red and, thus, has enhanced variability on long time scales.

Another important topic in climate research is the detection of trends. It is important to objectively identify trends which are caused by external forcings and are not due to sampling variability. The effect of sampling variability can be understood from the theory of stochastic processes. A first order Markov process is a stationary process and has no trends. On the other hand, a time series of finite length produced by a first order Markov process can have artificial or local "trends" (Wunsch, 1999; Feldstein, 2002b). Those 'trends' are not due to an intrinsic trend of the stochastic process but just due to insufficient sample size.

Another issue of trend identification is that in most studies evidence for a linear trend at the end of a time series is found by visually looking for the starting point of the trend. This practice was named "eyeballing" by Percival and Rothrock (2005). It is important to recognise that the definition of a trend should always be relative to a time scale; otherwise it is hard to distinguish between a "real" trend and a trend which is part of a low-frequency oscillation. An obvious example is the diurnal cycle of temperature. If one would look only at a $6 \mathrm{~h}$ snapshot of a typical day one would surely see a "trend"; but of course this local "trend" is part of the diurnal variation of temperature. The climate system has also (quasi)-periodic oscillations on longer time scales with the El Nino-Southern Oscillation phenomenon as the most pronounced example. It is important to associate trends with a certain time scale and, thus, to define trends over the whole available time series length. The EMD method provides a systematic way of identifying nonlinear trends in time series (Wu et al., 2007).

This study is structured as follows: Sect. 2 briefly explains the EMD method and the algorithm used, Sect. 3 describes the data used. In Sect. 4 we explain climate noise and investigate the climate noise characteristics of EMD. In Sect. 5 we present the results of the EMD analysis of the teleconnection indices and discuss them in Sect. 6 before we conclude our study in Sect. 7.

\section{Fundamentals of Empirical Mode Decomposition}

The Empirical Mode Decomposition (Huang et al., 1998; Huang and $\mathrm{Wu}, 2008$ ) is an algorithm to decompose a time series into a finite number of Intrinsic Mode Functions (IMF)

$x(t)=\sum_{j=1}^{M} \psi_{j}(t)+R(t)$

where the IMF $\psi_{j}$ can be written in polar coordinates

$\psi_{j}(t)=r_{j}(t) \sin \left(\theta_{j}(t)\right)$

where $r_{j}$ is the $j$-th amplitude, $\theta_{j}$ the $j$-th instantaneous frequency and $R$ the residual. Both, the amplitude and frequency are time-dependent. The transformation to Eq. (2) can be done by the Hilbert-Huang transform (Huang et al., 1998). As (2) shows, an IMF is different from Fourier modes where both $r_{j}$ and $\theta_{j}$ are time independent.

An IMF is defined by the following two properties (Huang et al., 1998)

- Each IMF $\psi_{j}$ has exactly one zero crossing between two consecutive local extrema.

- The local mean of each IMF $\psi_{j}$ is zero

The following algorithm estimates IMFs from a given time series (Huang et al., 1998):

1. Find all maxima and minima of the time series

2. Fit a cubic spline through all maxima (minima); these splines define the upper $e_{\text {up }}$ (lower $e_{\mathrm{lo}}$ ) envelope of the time series

3. Calculate mean of upper and lower envelope $m(t)=\frac{e_{\mathrm{up}}(t)+e_{\mathrm{lo}}(t)}{2} ;$ the resulting curve represents the first IMF

4. Subtract IMF from time series and go to 1 and repeat procedure until the residual is not an IMF anymore

In practice, the algorithm has to be refined by a so-called "sifting" process (e.g. Huang et al., 1998) which amounts to iterating steps 1 through 3 until this can be considered a zero mean to some stopping criterion. Once this is achieved the effective IMF has been determined. In this study we use the stopping criterion by Rilling et al. (2003). First let us define the mode amplitude

$a(t)=\frac{e_{\mathrm{up}}(t)-e_{\mathrm{lo}}(t)}{2}$

and the evaluation function

$\sigma(t)=\left|\frac{m(t)}{a(t)}\right|$

The sifting procedure is repeated till $\sigma(t)<\theta_{1}$ for a prescribed fraction of the time series length $(1-\alpha)$ and while 

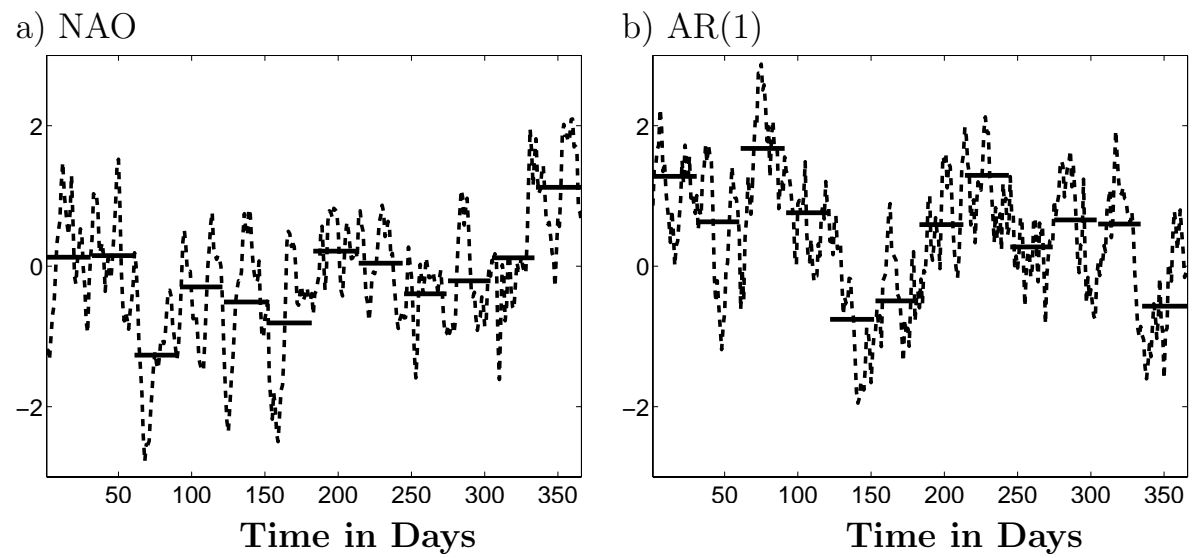

Fig. 1. Comparison of the (a) dimensionless daily NAO index and (b) a realisation of the corresponding AR(1) process (dashed lines) for a representative period of 366 days. The horizontal solid lines indicate monthly mean values derived from daily values.

$\sigma(t)<\theta_{2}$ for the remaining fraction. We use the values $\alpha=0.05, \theta_{1}=0.05$ and $\theta_{2}=0.5$ (Rilling et al., 2003). The results in this study are robust to the choice of these particular values.

The above algorithm is repeated until all IMFs are extracted and the residual is not an IMF anymore; thus, it violates the above two IMF criteria. The residual can now be interpreted as the instantaneous mean of the time series. In case this instantaneous mean is not constant we refer to it as a trend, which is possibly nonlinear (Wu et al., 2007) on the time scale of the time series length.

\section{Data}

In this study we use different indices of atmospheric teleconnection patterns which explain much of the variance in their respective hemisphere and influence surface weather and climate conditions (Wallace and Gutzler, 1981; Feldstein, 2000a; Thompson and Wallace, 2000a). Since one of the main aims of this study is to identify nonlinear trends we decided to use station-based indices for the NAO and SAM while the NP index is based on weather charts. These instrumental indices cover a longer time period than the currently available reanalysis data sets.

The NAO index is the Jones et al. (1997) monthly mean instrumental index for the period 1825 through 2007 (available from http://www.cru.uea.ac.uk/). It is based on the difference of standardised and homogenised monthly mean sea level pressure over Gibraltar and Southwest Iceland. It contains all months of the year. The NAO describes a large-scale see-saw pattern over the North Atlantic and has a strong impact on surface weather and climate conditions in Europe and the east coast of the US. Due to the normalization of the sea level pressure the NAO index is dimensionless and the an- nual cycle of the first two moments is effectively subtracted (this also applies to the other two used indices below).

The NP index is the normalized and area-weighted sea level pressure over the region $30^{\circ} \mathrm{N}-65^{\circ} \mathrm{N}, 160^{\circ} \mathrm{E}-140^{\circ} \mathrm{W}$ (available from http://www.cgd.ucar.edu/cas/jhurrell/ npindex.html) for the period 1899 through 2006 for all months of the year (Trenberth and Hurrell, 1994). This index is based on charts of sea level pressure. This pattern has a center of action which spans the central latitudes of the western and central North Pacific and centers of action of opposite and weaker polarity over eastern Siberia and Alaska and is closely related to the PNA.

The SAM index is defined as the difference of the normalised monthly and zonal mean sea level pressure at $40^{\circ} \mathrm{S}$ and $65^{\circ} \mathrm{S}$ from stations close to these two latitude bands (Marshall, 2003) (available from http://www.nerc-bas.ac.uk/ icd/gjma/sam.html) for the period 1957 through 2007. The SAM has a strong zonally symmetric geographic dipole structure in the mid- and high-latitudes.

In order to carry out significance tests of the IMFs against a climate noise hypothesis we need daily indices of the above teleconnection patterns. The daily data is needed to fit an AR(1) model, which we use as our paradigmatic climate noise model. The AR(1) model is a simple linear stochastic model which produces a red power spectrum which is typical for teleconnection indices (e.g. Wunsch, 1999). On the other hand, an AR(1) model will fail in reproducing the statistical characteristics for systems which are nonlinear and/or exhibit a trend. Thus, the AR(1) model constitutes a reasonable choice as a null model in our study.

Currently, the above indices are not available as daily indices due to missing data. Therefore, we derive daily teleconnection indices from the daily National Centers for Prediction-National Center for Atmospheric Research (NCEP-NCAR) reanalysis sea level pressure data set for the 
a) IMF-1

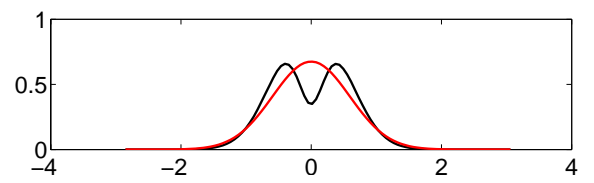

b) IMF-2

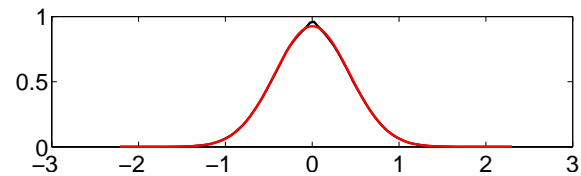

c) IMF-3

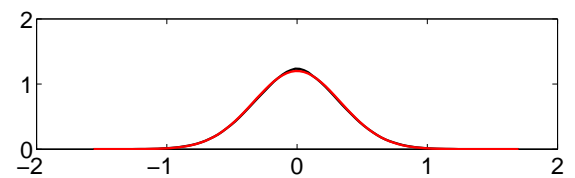

d) $\mathrm{IMF}-4$

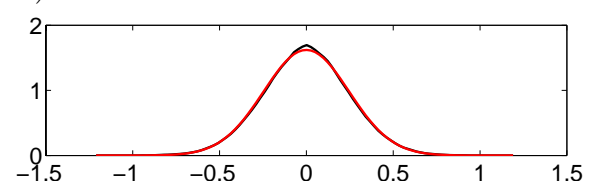

e) $\mathrm{IMF}-5$

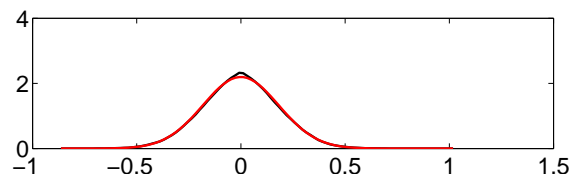

f) IMF-6

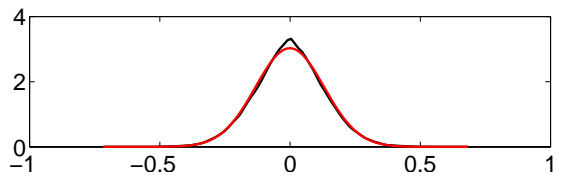

g) IMF-7

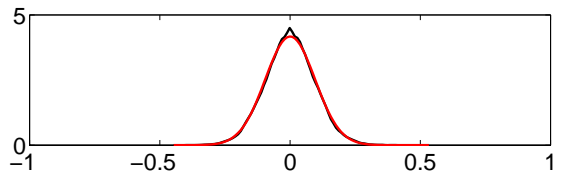

h) $\mathrm{IMF}-8$

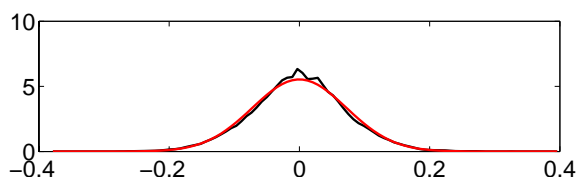

Fig. 2. Probability Density Functions of IMFs for a AR(1) process (black line) and fitted theoretical Normal distributions (red line).

period 1948 through 2007. For this purpose we first calculate the linear regression of the respective monthly mean index onto monthly mean sea level pressure. Then we use the regression patterns to calculate daily teleconnection indices by projecting the regression patterns onto daily sea level pressure fields.

\section{Climate noise characteristics of EMD}

Climate noise is that part of variability which stems from averaging of time series whose intrinsic time scale is shorter than the averaging period. Most studies of atmospheric climate variability use monthly or seasonally averaged data to study climate variability. However with the typical time scale associated with major atmospherically relevant processes is on the order of 5-10 days, e.g. associated with synoptic weather systems and teleconnection patterns (Feldstein, 2000a, 2002a, 2003; Cash and Lee, 2001; Franzke and Feldstein, 2005). Hence, averaged time series of finite length which are produced by a stationary process with an intrinsic time scale smaller than the averaging period can exhibit variability on longer time scales and even trends. Thus, it is important to examine if observed climate variability and trends are significantly different from climate noise.

An autoregressive process of first order (AR(1); von Storch and Zwiers, 1999) can be utilized as a null hypothesis to test for climate noise. The general visual similarity of such a process to observed data is depicted in Fig. 1 where a representative sequence of a daily NAO index and a realisation of the corresponding AR(1) process are displayed together with the respective monthly means. Both time series appear to undergo variations on relatively long time scales. Thus, it is possible that climate noise contributes to the lowfrequency variability of averaged time series and in the following we want to test if the IMFs can be distinguished from climate noise. Previously, Wu and Huang (2004) investigated the EMD characteristics of uniformly distributed noise and found that EMD is a dyadic filter bank and that all IMFs of white noise are Gaussian distributed. Because an $\mathrm{AR}(1)$ process constitutes a much better fit to geophysical data than white noise, we examine first its EMD characteristics. For this purpose we estimate the parameters of an $\mathrm{AR}(1)$ processes from the daily NAO, NP and SAM time series and then we generate for each AR(1) model of the corresponding teleconnection index an ensemble of 1000 realisations. These realizations are then averaged to derive the corresponding monthly mean surrogate time series. An AR(1) process is defined as (von Storch and Zwiers, 1999)

$x_{t+1}=\alpha x_{t}+\sigma \zeta_{t}$

Its autoregression coefficient is

$\alpha=\frac{<x_{t} x_{t+1}>}{\operatorname{Var}(x)}$

and noise variance

$\sigma^{2}=\frac{\operatorname{Var}(x)}{1-\alpha^{2}}$. 


\section{a) IMF-1}

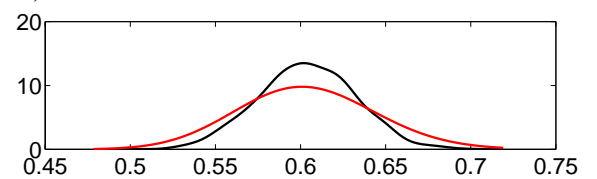

b) IMF-2

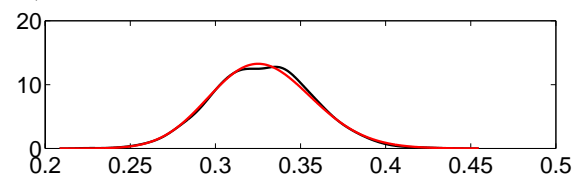

c) IMF-3

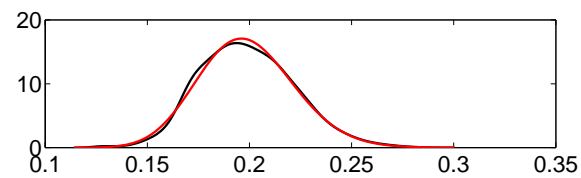

d) IMF-4

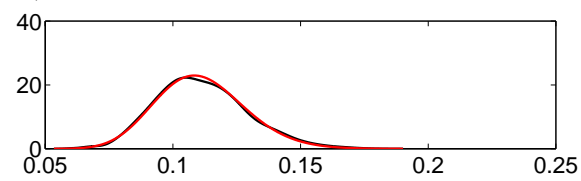

e) IMF-5

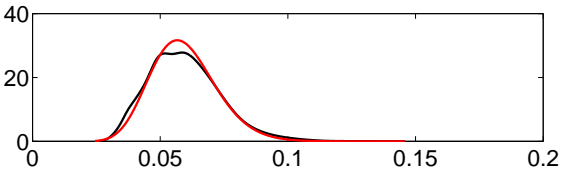

f) IMF-6

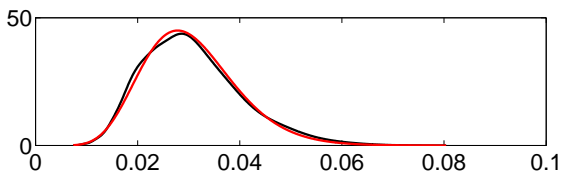

g) IMF-7

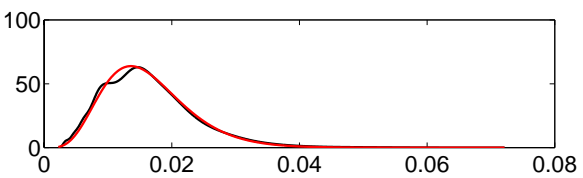

h) IMF-8

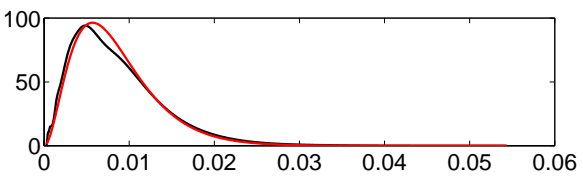

Fig. 3. Probability Density Functions of the variance spread of IMFs for an averaged AR(1) process (black line) and fitted theoretical $\chi^{2}$ distributions (red line).

Table 1. Mean period in months of NAO and corresponding AR(1) process.

\begin{tabular}{ccccccccccc}
\hline & IMF1 & IMF2 & IMF3 & IMF4 & IMF5 & IMF6 & IMF7 & IMF8 & IMF9 & IMF10 \\
\hline NAO & 2 & 6 & 12 & 22 & 44 & 82 & 170 & 366 & 628 & 2196 \\
AR(1) & 2 & 6 & 12 & 24 & 50 & 102 & 212 & 456 & 1152 & 2196 \\
\hline
\end{tabular}

Both coefficients can be estimated from a given time series $x$ (with the mean subtracted) and amount to estimating the variance and the lag- 1 autocorrelation function value. The symbol $\zeta$ denotes a random variable from a Gaussian distribution with zero mean and unit variance. Because an AR(1) process is a linear process driven by Gaussian white noise it has purely Gaussian statistics.

An investigation with EMD of these realisations reveals that the mean period approximately doubles for consecutive IMFs (Tables 1, 2, 3) and, thus, confirms that EMD is a dyadic filter bank. An investigation of the Probability Density Functions (PDF) reveals that all individual IMF components $\left(\psi_{j}(t)\right)$ are Gaussian distributed except for the first which shows a bimodal distribution (Fig. 2). The Gaussian fits are less good for higher IMFs. This is because the higher IMF components describe the low-frequency behaviour and, thus, contain a smaller number of oscillations and the number of events decreases and the distributions become less smooth. The fits get better when a longer time series is used (not shown). From this we can deduce and also numerically verify that the variance of all individual IMF components $\left(\psi_{j}^{2}(t)\right)$ are $\chi^{2}$ distributed (not shown).

Next we investigate the probability distribution of the variance contained in IMF components from an ensemble of $1000 \mathrm{AR}(1)$ realisations $p\left(\left(\sum_{t} \psi_{j}^{2}(t)\right)_{\text {Ensemble }}\right)$. These distributions are nearly $\chi^{2}$ distributed (Fig. 3). Figure 3 shows that most PDFs of the variance spread of IMFs are very good fits to the theoretical $\chi^{2}$ distributions; though the fit is less good for IMF1. Following the arguments of Wu and Huang (2004) for white noise we estimate the number of degrees of freedom of the $\chi^{2}$ distributions as the mean energy of the IMF times the time series length. This is verified due to the good fit of the distribution when compared with the theoretical $\chi^{2}$ distributions (Fig. 3). By utilizing the arguments of Wu and Huang (2004) we can now assign statistical significance of the information content for the IMFs from climate noise. We will use this result to test for the significance of the IMFs and trends in the teleconnection indices in the next section. 
Table 2. Mean period in months of NP and corresponding AR(1) process.

\begin{tabular}{cccccccccc}
\hline & IMF1 & IMF2 & IMF3 & IMF4 & IMF5 & IMF6 & IMF7 & IMF8 & IMF9 \\
\hline NP & 4 & 8 & 14 & 26 & 52 & 86 & 216 & 650 & 866 \\
AR(1) & 4 & 6 & 14 & 26 & 52 & 108 & 232 & 534 & 1296
\end{tabular}

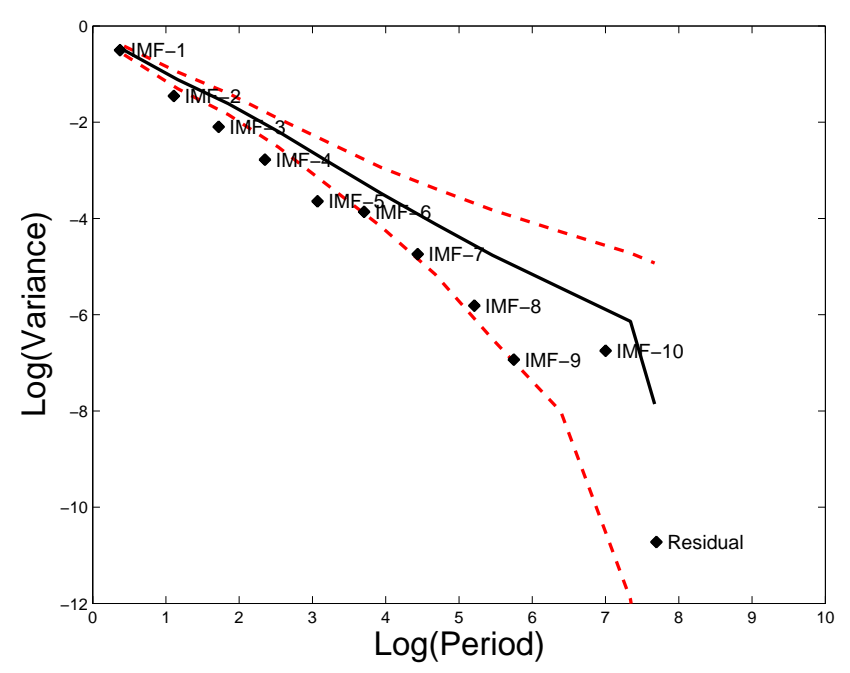

Fig. 4. Statistical significance test for the NAO. The time scale of the residual trend is set to the length of the time series. The solid black line is the mean variance of the AR(1) ensemble and the dashed red lines denote the $2.5 \%$ and $97.5 \%$ percentiles. The axes are scaled by the natural logarithm.

Table 3. Mean period in months of SAM and corresponding AR(1) process.

\begin{tabular}{cccccccc}
\hline & IMF1 & IMF2 & IMF3 & IMF4 & IMF5 & IMF6 & IMF7 \\
\hline SAM & 4 & 6 & 14 & 26 & 56 & 154 & 244 \\
AR(1) & 4 & 6 & 14 & 30 & 60 & 128 & 318 \\
\hline
\end{tabular}

In summary, the results of the characteristics of averaged AR(1) processes using the EMD method share many similarities with those of white noise (Wu and Huang, 2004). One of the main differences is that the relationship between the logarithm of the variance and the logarithm of the averaged period differs from that of white noise. This linear relationship is for white noise (Wu and Huang, 2004)

$\ln E_{j}=-\ln T_{j}$

where $E$ denotes the variance of the $j$ th IMF and $T$ its averaged period. For an averaged AR(1) process this relationship is

$$
\ln E_{j}=-a \ln T_{j}
$$

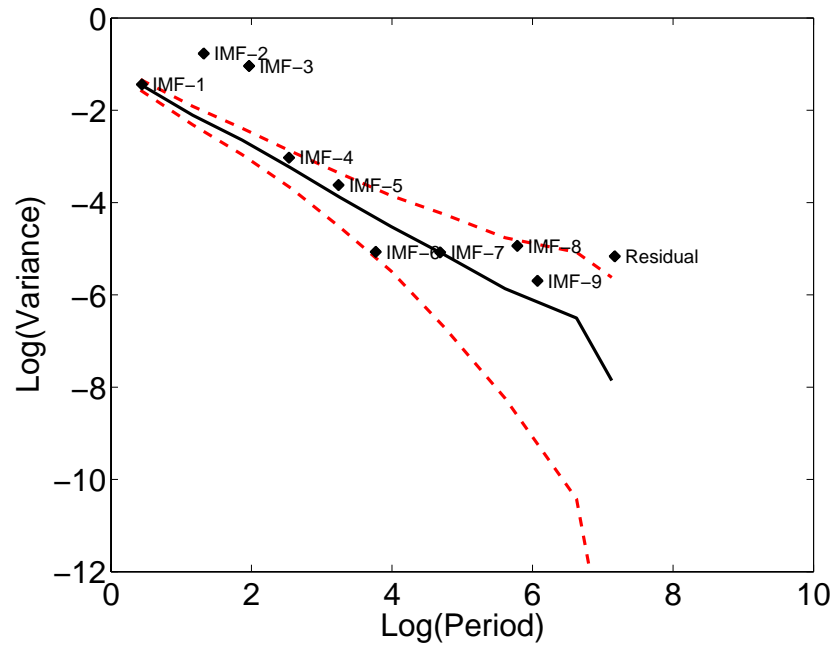

Fig. 5. Statistical significance test for the NP. The time scale of the residual trend is set to the length of the time series. The solid black line is the mean variance of the AR(1) ensemble and the dashed red lines denote the $2.5 \%$ and $97.5 \%$ percentiles. The axes are scaled by the natural logarithm.

where $-a$ denotes the slope, which is in general different from -1 . The slopes are -1.8 for AR(1) processes fitted to the NAO and NP indices and -2.1 for the SAM index (Figs. 4, 5 and 6). The effect of this difference is that the $j$-th IMF component of white noise and averaged AR(1) can have different variance and this in turn will change the number of degrees of freedom. The change in number of degrees of freedom will influence whether a IMF component is significant or not. Because most climatic time series are temporally correlated using an averaged AR(1) model as a climate noise null hypothesis is much more appropriate in a geophysical setting. It has to be noted, that depending on the underlying time scale of the Markov process climate noise can effectively be white noise. This can occur if the time scale of the Markov process is much shorter than the averaging period.

\section{Results}

In this section we describe the results of applying the EMD method to atmospheric teleconnection indices and testing the significance of the IMFs and trends. 
The IMFs for the NAO, NP and SAM indices are displayed in Figs. 7, 8 and 9. For all three teleconnection indices the first few IMFs explain much more variance than the trailing IMFs. This suggests that most of the variance is due to intraannual processes since these IMFs have mean periods of a few months (Tables 1,2 and 3). In the case of the NAO and SAM IMF1 captures most of the variance, while the third IMF explains most of the variance for the NP index. For all three teleconnection indices the residual shows marked differences to a linear least-squares fit, which is usually utilized to detrend a time series and, thus, can introduce an error.

The statistical significance of the IMFs is tested against a climate noise null hypothesis represented by an averaged AR(1) model. For this test we generate 1000 realisations of the AR(1) model corresponding to the respective teleconnection index and then calculate monthly averages. Then we apply EMD to this averaged time series. To display the results we plot the logarithm of the mean variance and the $2.5 \%$ and 97.5\% percentiles of the IMF variance distribution against the logarithm of the mean period (Figs. 4, 5 and 6). We have chosen to use a non-parametric approach by using the percentiles estimated from the directly measured PDFs of the variance distribution instead of the theoretical $\chi^{2}$ distribution percentiles. Though the differences between both approaches are very small (not shown). If the variance of an IMF of the respective teleconnection index lies outside of either of the two percentiles then the IMF is significantly different from climate noise. The logarithm of the mean variance of the IMFs of climate noise shows roughly a linear relationship with the logarithm of the mean period. But the slope of this linear relationship is different from that of white noise $(\mathrm{Wu}$ and Huang, 2004). The slope is -1 in the case of white noise (Wu and Huang, 2004) whereas it is -1.8 for both the NAO and NP indices and -2.1 for the SAM index. This difference in the slope has direct implication for the significance test, since the number of degrees of freedom are proportional to the the variance. This means that using a white noise instead of an averaged AR(1) null hypothesis will lead to different IMF modes being significant or insignificant.

The climate noise test reveals that the significant modes are IMF 2 through 5 for the NAO, IMF 2 and 3 and the residual for the NP index and IMF 2, 3, 7 and the residual for the SAM (Figs. 4, 5 and 6). Thus, the NAO has no significant trend over the period 1825-2007 which can be distinguished from artificial trends expected from a first order Markov process, if the residual is interpreted as a trend. The NP index and SAM have trends which are significantly different from artificial trends expected from an AR(1) process. The NP index has a trend towards its negative phase, while the SAM has a trend towards its positive phase. A comparison of the trends identified by EMD with straightforward linear leastsquares fits to the respective index time series shows that the trends are nonlinear in the sense that they are better described by a higher order polynomial than a straight line and that the trends of both the NP and SAM indices weaken consider-

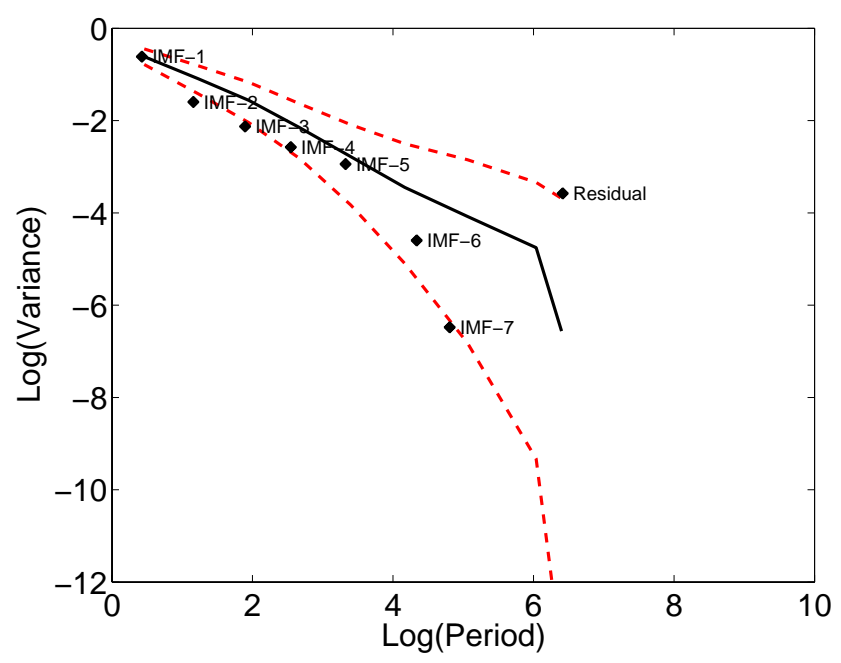

Fig. 6. Statistical significance test for the SAM. The time scale of the residual trend is set to the length of the time series. The solid black line is the mean variance of the AR(1) ensemble and the dashed red lines denote the $2.5 \%$ and $97.5 \%$ percentiles. The axes are scaled by the natural logarithm.

ably over the last few decades (Figs. 8k and 9i). This further shows that detrending an index time series with a linear leastsquares fit is likely to introduce a sizeable error in any further analysis of the time series.

Our results also suggest that most of the interannual and decadal variability of the teleconnection indices cannot be distinguished from a climate noise process. The significant IMFs at the 5\% level of the NAO have mean periods between 6 and 44 months, the NP 8 and 14 months and the SAM 6, 14 and 244 months. Thus, most of the significant IMFs have mean periods which are on the intraannual up to annual time scale and only the NAO has a significant IMF mode with periods up to 44 months on an interannual time scale. The SAM is the only index which also shows a significant signal on a decadal time scale.

The sum of the explained variance of the significant IMFs gives a rough measure of the signal-to-noise ratio. By this measure $40.9 \%$ of the NAO, $70.5 \%$ of the NP and $33.4 \%$ of the SAM variability cannot be accounted for as being climate noise. Thus, the NP index is the index which contains the most information content.

\section{Discussion}

Previous studies have investigated whether atmospheric teleconnection indices contain significant trends. One of the most discussed is the NAO index. Many studies used linear methods to identify trends in the NAO (e.g. Hurrell, 1995; Thompson et al., 2000b; Feldstein, 2002b). By using the EMD method and the climate noise test we do not find a 
a) $\mathrm{NAO}$

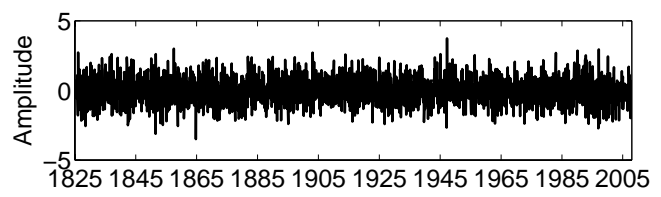

b) IMF-1 $(55.7 \%)$

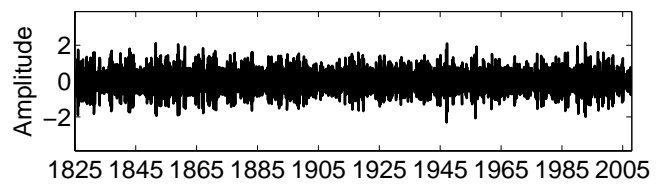

c) $\operatorname{IMF}-2(21.5 \%)$

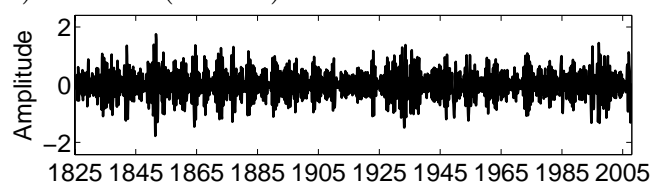

d) IMF-3 (11.3\%)

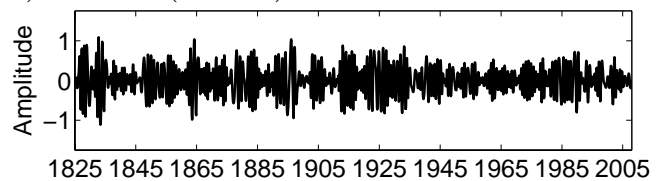

e) IMF-4 (5.7\%)

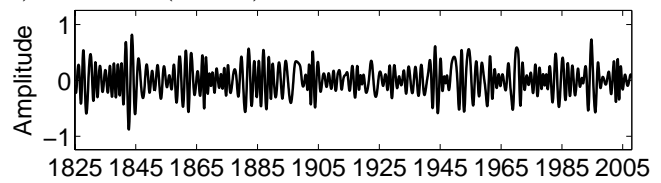

f) $\operatorname{IMF}-5(2.4 \%)$

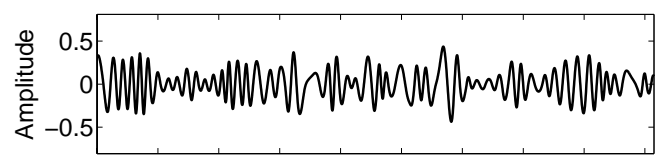

1825184518651885190519251945196519852005

Year g) IMF-6 (1.9\%)

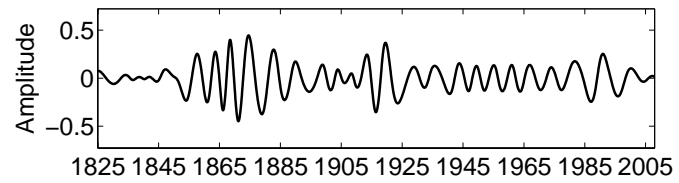

h) IMF-7 (0.8\%)

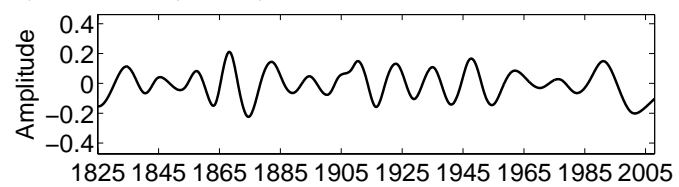

i) $\mathrm{IMF}-8(0.3 \%)$

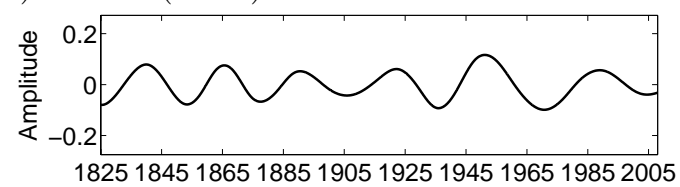

j) IMF-9 $(0.1 \%)$

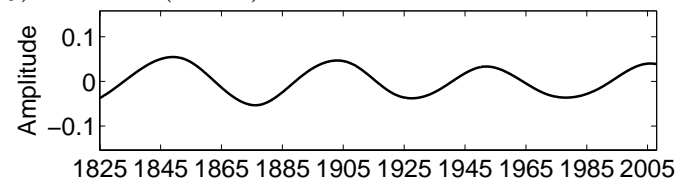

k) $\operatorname{IMF}-10(0.1 \%)$

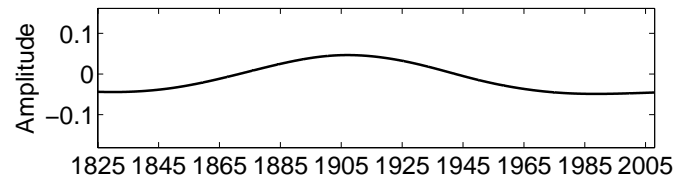

l) Residual

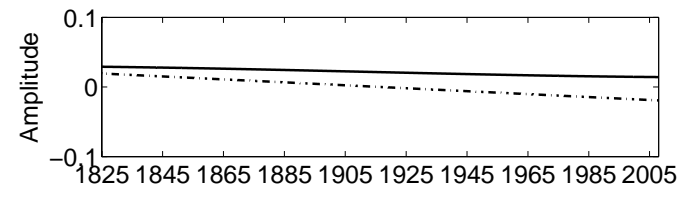

Year

Fig. 7. Intrinsic Mode Functions and explained variances of the Empirical Mode Decomposition for the monthly NAO index for the years 1825-2007. (a) NAO index, (b-k) IMFs and (l) residual. The residual has the least-squares linear trend of the NAO superimposed (dotdashed line).

significant trend. Our results confirm the studies by Wunsch (1999) and Percival and Rothrock (2005) who also did not find a significant trend in the NAO, while Hurrell (1995) and Thompson et al. (2000b) found trends by using a linear leastsquares estimator but did not test against a noise model. This study also finds evidence of a significant trend in the SAM index towards its positive phase, in agreement with the conclusion by Marshall (2003) based on a linear least-squares fit. However, it has to be noted that the trend identified with EMD is nonlinear and the trend attenuates during the last few years (Fig. 9i). Thus, a linear least-squares fit provides a less good fit and might introduce errors in subsequent analysis of a detrended SAM index. It is interesting to note, that the here found attenuation of the SAM trend occurs at the same time that the ozone hole is recovering. Recent studies suggest that the preference of the positive SAM phase is connected to the ozone hole and also, but to a lesser extend, to the increasing greenhouse gas concentrations (e.g. Roscoe and Haigh, 2007; Perlwitz et al., 2008). To the authors knowledge this is the first time that a trend in the NP index is discussed.

Another interesting result is that most of the variability which is different from climate noise is intraannual variability. This is consistent with the climate noise paradigm by Leith (1973), Madden (1981), Feldstein (2000a) and Czaja 
a) NP

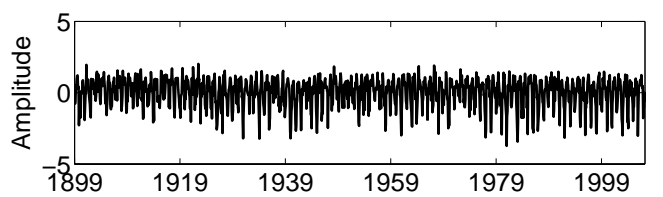

b) IMF-1 $(20.4 \%)$

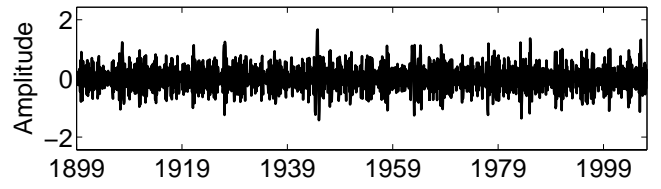

c) IMF-2 $(40.0 \%)$

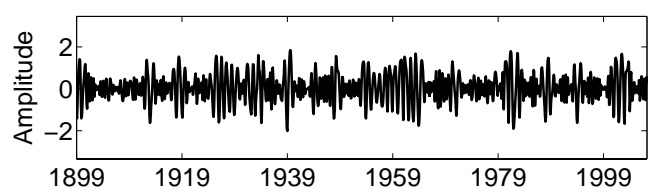

d) IMF-3 $(30.5 \%)$

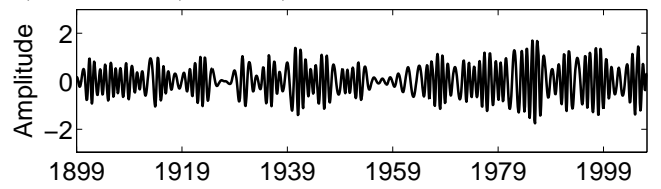

e) IMF-4 (4.2\%)

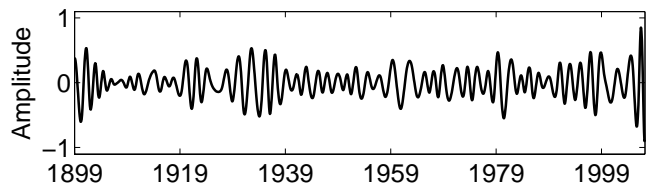

f) $\operatorname{IMF}-5(2.3 \%)$

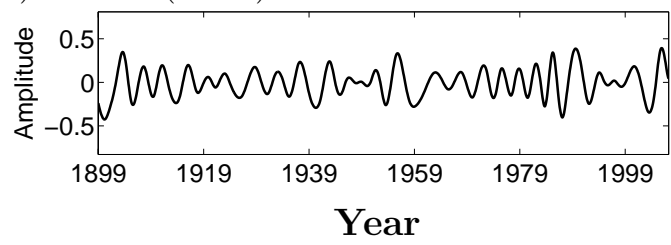

g) IMF-6 $(0.5 \%)$

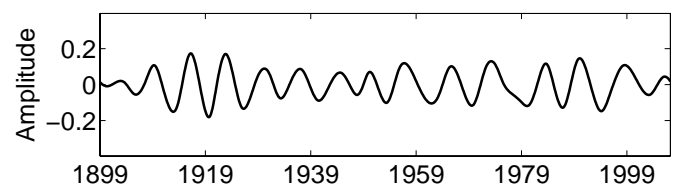

h) $\operatorname{IMF}-7(0.5 \%)$

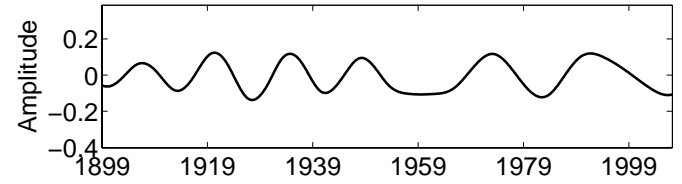

i) $\operatorname{IMF}-8(0.6 \%)$

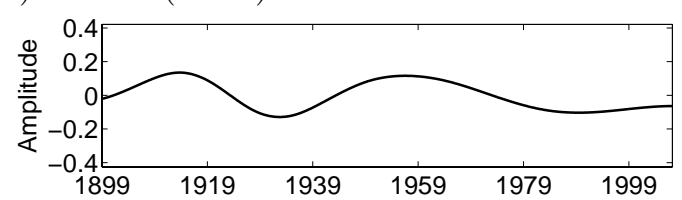

j) IMF-9 (0.3\%)

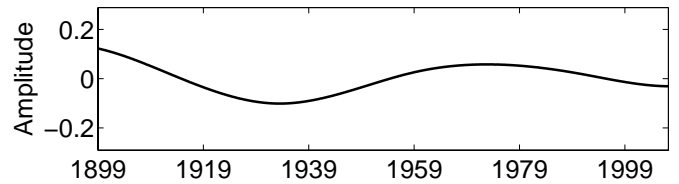

k) Residual

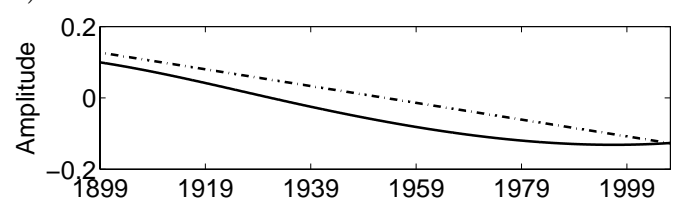

Fig. 8. Intrinsic Mode Functions and explained variances of the Empirical Mode Decomposition for the monthly NP index for the years 1899-2006. (a) NP index, (b-j) IMFs and (k) residual. The residual has the least-squares linear trend of the NP superimposed (dot-dashed line).

et al. (2003). This paradigm suggests that apparent interannual and longer time scale fluctuations of the teleconnection indices can arise due to energetic atmospheric fluctuations on time scales of days to a few weeks. This is also consistent with the findings of Feldstein (2000a, 2002a, 2003) and Franzke and Feldstein (2005) that Northern Hemisphere teleconnection patterns have an intrinsic time scale of about 10 days and are a largely atmospheric phenomenon. This suggests that rather short time scale processes associated with synoptic scale waves are able to produce apparent variability on a much longer time scale, thus producing the multi-scale appearance of teleconnection indices. Furthermore, this re- sult has possible implications for predictability. It suggests that for the NP only intraannual variability has predictive skill beyond that expected of climate noise. While our results suggest that the NAO might have predictive skill for up to 44 months and the SAM for 244 months beyond that of climate noise. The climate noise paradigm does not rule out that interactions with other climate subsystems like the ocean or nonlinear atmospheric processes cause the variability of the teleconnection indices on longer time scales. It only means that a simple stochastic process is sufficient to describe this variability and, thus might limit the predictability. 
a) SAM

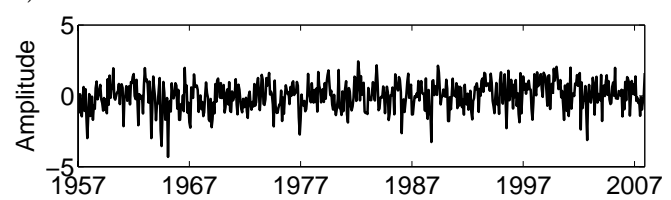

b) IMF-1 $(51.9 \%)$

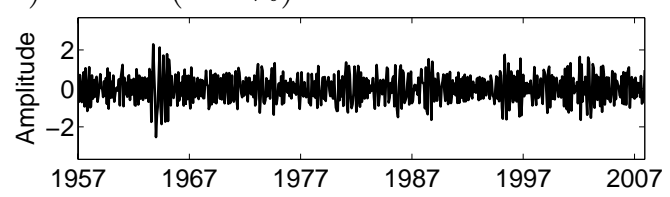

c) IMF-2 $(19.4 \%)$

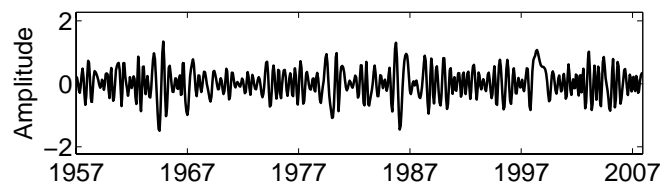

d) IMF-3 (12.7\%)

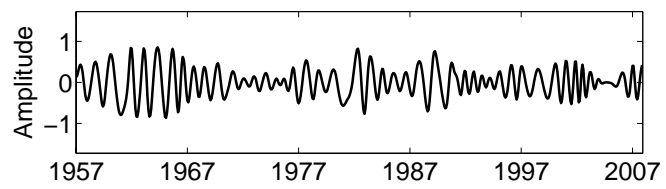

e) IMF-4 $(7.0 \%)$

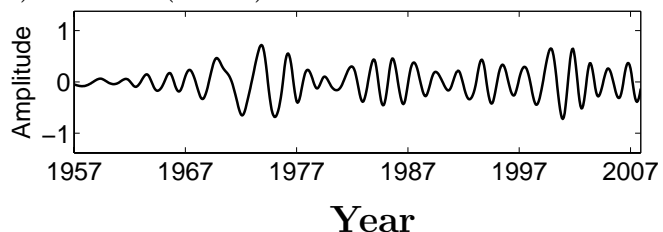

f) $\operatorname{IMF}-5(4.7 \%)$

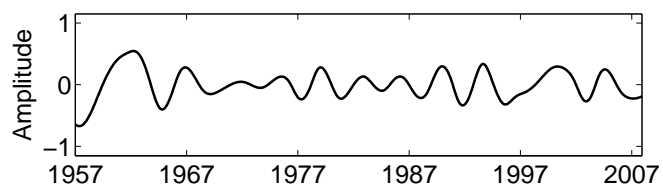

g) IMF-6 $(1.5 \%)$

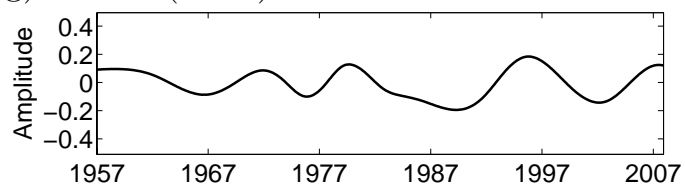

h) IMF-7 (1.3\%)

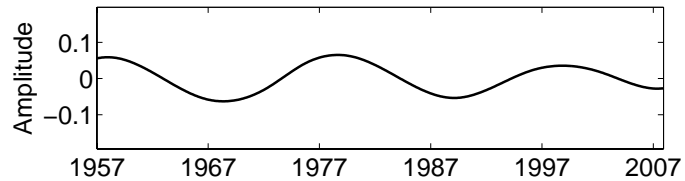

i) Residual

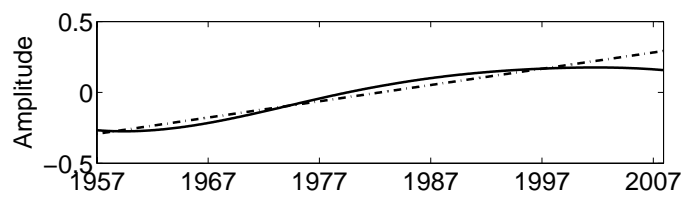

Year

Fig. 9. Intrinsic Mode Functions and explained variances of the Empirical Mode Decomposition for the monthly SAM index for the years 1957-2007. (a) SAM index, (b-h) IMFs and (i) residual. The residual has the least-squares linear trend of the SAM superimposed (dotdashed line).

The ability to identify nonlinear trends should prove useful in low-order stochastic modeling of climate indices (e.g. Crommelin and Vanden-Eijnden, 2006; Majda et al., 2008), since subtracting a linear least-squares trend might change the characteristics of important quantities like the decay of the autocorrelation function and the PDF's. The EMD method could be also used as a pre-filter where only the significant IMF's are used for fitting of low-order dynamics and the insignificant IMF's are treated as a red noise stochastic forcing.

\section{Conclusions}

In this study we used the EMD method to investigate the climate noise properties and the existence of nonlinear trends of three important teleconnection indices: the NAO, NP and SAM. For this purpose we first examined the climate noise characteristics of the EMD method. We used a simple red noise model as a null hypothesis to test the statistical significance of IMFs of the three atmospheric teleconnection indices. Our results are

- EMD acts effectively as a dyadic filter bank and the IMFs of climate noise are nearly Gaussian distributed (except for IMF1)

- The spread of the variances of climate noise IMFs are nearly $\chi^{2}$ distributed

- Most of the significant variability of the analysed teleconnection indices occurs on intraannual and annual time scales, while most of the interannual and decadal variability cannot be distinguished from climate noise

- The SAM is the only index with a significant signal on a decadal time scale 
- The NP and SAM teleconnection indices have significant nonlinear trends

- The NAO has no significant trend

Our results show that the EMD method together with the climate noise test presents a powerful tool to investigate climate variability and to objectively detect nonlinear trends. Our results also suggest that detrending a time series by subtracting a linear least-squares fit is not necessarily appropriate.

Acknowledgements. I would like to thank P. Flandrin for freely providing the code for EMD (available at: http://perso.ens-lyon.fr/patrick.flandrin/emd.html) and M. Freeman and N. Watkins for a very careful reading of an earlier version of this manuscript.

Edited by: J. Kurths

Reviewed by: one anonymous referee

\section{References}

Bengtsson, L., Hagemann, S., and Hodges, K. I.: Can climate trends be calculated from reanalysis data?, J. Geophys. Res., 109, D11111, doi:10.1029/2004JD004536.

Cash, B. A. and Lee, S.: Observed Nonmodal Growth of the PacificNorth American Teleconnection Pattern, J. Climate, 14, 10171028, 2001.

Crommelin, D. T. and Vanden-Eijnden, E.: Reconstruction of Diffusions using spectral data from timeseries, Comm. Math. Sci., 4, 651-668, 2006.

Czaja, A., Robertson, A. W., and Huck, T.: The role of Atlantic Ocean-Atmosphere coupling in affecting North Atlantic Oscillation variability, in: The North Atlantic Oscillation: Climatic Significance and Environmental Impact, edited by: Hurrell, J. W., Kushnir, Y., Ottersen, G., and Visbeck, M., Geophysical Monograpgh 134, American Geophysical Union, 147-172, 2003.

Duffy, D. G.: The Application of Hilbert-Huang Transforms to Meteorological Datasets, J. Atmos. Oceanic Technol., 21, 599-611, 2004.

Feldstein, S. B.: The Timescale, Power Spectra, and Climate Noise Properties of Teleconnection Patterns, J. Climate, 13, 44304440, 2000a.

Feldstein, S. B.: Is Interannual Zonal Mean Flow Variability Simply Climate Noise?, J. Climate, 13, 2356-2362, 2000b.

Feldstein, S. B.: Fundamental Mechanisms of the growth and decay of the PNA teleconnection pattern, Q. J. Roy. Meteorol. Soc., 128, 775-796, 2002a.

Feldstein, S. B.: The Recent Trend and Variability Increase of the Annular Mode, J. Climate, 15, 88-94, 2002b.

Feldstein, S. B.: The dynamics of the NAO teleconnection pattern growth and decay, Q. J. Roy. Meteorol. Soc., 129, 901-924, 2003.

Franzke, C. and Feldstein, S. B.: The Continuum and Dynamics of Northern Hemisphere Teleconnection Patterns, J. Atmos. Sci., 62, 3250-3267, 2005.

Hasselmann, K.: Stochastic Climate Models. Part I. Theory, Tellus, 28, 473-484, 1976.
Huang, N. E., Shen, Z., Long, S. R., Wu, M. C., Shih, H. H., Zheng, Q., Yen, N.-C., Tung, C. C., and Liu, H. H.: The Empirical Mode Decomposition and the Hilbert Spectrum for Nonlinear and NonStationary Time Series Analysis, Proc. R. Soc. Lond. A, 454, 903-995, 1998.

Huang, N. E., Shen, Z., and Long, S. R.: A New View of Nonlinear Water Waves: The Hilbert Spectrum, Ann. Rev. Fluid Mech., 31, 417-457, 1999.

Huang, N. E., Wu, M.-L. C., Long, S. R., Shen, S. S. P., Qu, W., Gloersen, P., and Fan, K. L.: A Confidence Limit for the Empirical Mode Decomposition and Hilbert Spectral Analysis, Proc. R. Soc. Lond. A, 459, 2317-2345, 2003.

Huang, N. E. and Wu, Z.: A Review on Hilbert-Huang Transform: Method and its Applications to Geophysical Studies, Rev. Geophys., 46, 2008. RG2006, doi:10.1029/2007RG000228, 2008.

Hurrell, J. W.: Decadal Trends in the North Atlantic Oscillation and Relationships to Regional Temperature and Precipitation, Science, 269, 676-679, 1995.

Jones, P. D., Jonsson, T., and Wheeler, D.: Extension to the North Atlantic Oscillation using early instrumental pressure observations from Gibraltar and South-East Iceland, Int. J. Climatol., 17, 1433-1450, 1997.

Leith, C. E.: The Standard Error of Time-Average Estimates of Climatic Means, J. Appl. Meteorol., 12, 1066-1069, 1973.

Madden, R. A.: Estimates of the Natural Variability of TimeAveraged Sea-Level Pressure, Mon. Weather Rev., 104, 942952, 1976.

Madden, R. A.: A Quantitative Approach to Long-Range Prediction, J. Geophys. Res., 86, 9817-9825, 1981.

Majda, A. J., Franzke, C., and Khouider, B.: An applied mathematics perspective on stochastic modelling for climate, Phil. Trans. R., Soc. A, 366, 2429-2455, doi.10.1098/rsta.2008.0012, 2008.

Marshall, G. J.: Trends in the Southern Annular Mode from Observations and Reanalyses, J. Climate, 16, 4134-4143, 2003.

McDonald, A. J., Baumgaertner, A. J. G., Fraser, G. J., George, S. E., and Marsh, S.: Empirical Mode Decomposition of the atmospheric wave field, Ann. Geophys., 25, 375-384, 2007, http://www.ann-geophys.net/25/375/2007/.

Percival, D. B. and Rothrock, D. A.: 'Eyeballing' trends in climate time series: A cautionary note, J. Climate, 18, 886-891, 2005.

Perlwitz, J., Pawson, S., Fogt, R. L., Nielsen, J. E., and Neff, W. D.: Impact of stratospheric ozone hole recovery on Antarctic climate, Geophys. Res. Lett., 35, L08714, doi:10.1029/2008GL033317, 2008.

Rilling, G., Flandrin, P., and Goncalves, P.: On Empirical Mode Decomposition and Its Algorithms, IEEE-EURASIP Workshop on Nonlinear Signal and Image Processing NSIP-03, GRADO(I), 2003.

Roscoe, H. K. and Haigh, J. D.: Influences of ozone depletion, the solar cycle and the QBO on the Southern Annular Mode, Q. J. Roy. Meteorol. Soc., 133, 1855-1864, 2007.

Thompson, D. W. J. and Wallace, J. M.: Annular Modes in the Extratropical Circulation: Part I. Month-to-Month Variability, J. Climate, 13, 1000-1016, 2000a.

Thompson, D. W. J., Wallace, J. M., and Hegerl, G. C.: Annular Modes in the Extratropical Circulation: Part II. Trends, J. Climate, 13, 1017-1036, 2000b.

Trenberth, K. E. and Hurrell, J. W.: Decadal Atmosphere-Ocean Variations in the Pacific, Clim. Dynam., 9, 303-319, 1994. 
von Storch, H. and Zwiers, F., W.: Statistical Analysis in Climate Research, Cambridge University Press, New York, 484 pp., 1999.

Wallace, J. M. and Gutzler, D. S.: Teleconnections in the Geopotential Height Field during the Northern Hemisphere Winter, Mon. Rev. Weather, 109, 784-812, 1981.

Wu. Z. and Huang, N. E.: A Study of the Characteristics of White Noise using the Empirical Mode Decomposition Method, Proc. R. Soc. Lond. A, 460, 1597-1611, 2004.
Wu, Z., Huang, N. E., Long, S. R., and Peng, C. K.: On the Trend, Detrending, and Variability of Nonlinear and Nonstationary Time Series, Proc. Nat. Acad. Sci. USA, 104, 14889-14894, 2007.

Wunsch, C.: The Interpretation of Short Climate Records, with Comments on the North Atlantic and Southern Oscillations, B. Am. Meteorol. Soc., 80, 245-255, 1999. 